\title{
Towards synthetic enzymes
}

\section{Leonard F. Lindoy}

THE design and synthesis of molecules that might mimic the recognition and catalytic behaviour of enzymes is attracting more and more attention from chemists. Last month, Mary McPartlin and co-workers ${ }^{1}$ reported the X-ray structure of one such supramolecular system - the tris(pyridine) adduct of the zinc porphyrin cyclic trimer (1). Earlier work had shown ${ }^{2}$ that 1 is able to bind two suitable substrates in such a way that reaction between them is enhanced (in parallel to the behaviour of many enzymes). The promise of such research is that it will deliver new chemical processes that are both more energy-efficient and more specific in terms of the products formed than existing processes.

The X-ray structure confirms that there is room for two or three medium-sized guest molecules in the central cavity of $\mathbf{1}$, in accord with earlier predictions based on space-filling models and molecular

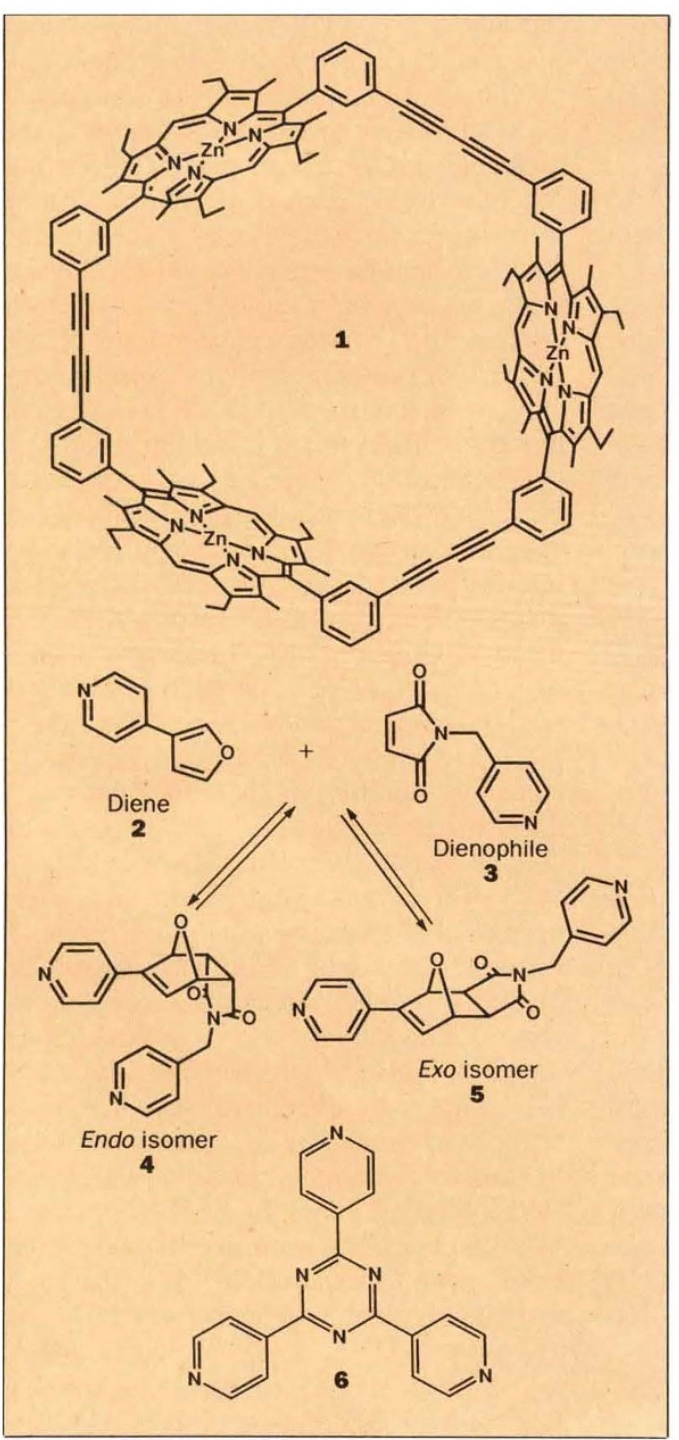

mechanics calculations. An unusual interpenetration of pairs of cyclic trimers occurs: one porphyrin of each trimer is a guest in the cavity of the other. The result is an interlocked 'dimer of trimers'. The flexibility of the individual cyclic trimers is demonstrated by their marked deviation from 3-fold symmetry in this solidstate structure. For achieving optimum host-guest formation, such flexibility may prove important in allowing the cavity to respond to the steric demands of different substrates.

The authors believe that the observed dimer formation in the solid will not be of much significance in solution; it will have little influence on the ability of 1 to incorporate suitable substrates and hence promote their reaction. Indeed, a preliminary X-ray study shows that similar dimer formation in the solid is absent in the corresponding tris(quinuclidine) adduct, even though it was crystallized under identical conditions to the tris(pyridine) adduct.

In progress towards a 'synthetic enzyme', Sanders and coworkers ${ }^{2}$ have demonstrated that 1 binds the diene 2 and the dienophile $\mathbf{3}$ in its cavity in such a way as to promote a Diels-Alder reaction between them. The reaction between the bound substrates proceeds at an initial rate some 6,000 times faster than for the equivalent pair in the absence of 1 . As in enzyme reactions, only one stereoisomer (the exo form) of the possible endo (4) and exo (5) products was detected at any stage during the reaction. In the absence of 1 , a mixture of both forms is produced, although the exo form is thermodynamically favoured (and is the sole product at the completion of the reaction).

There is evidence that each of the substrates 2 and 3 bind by means of their pyridyl nitrogens to adjacent zinc atoms of the same molecule of the cyclic trimer 1 . In this manner the respective reactive sites of $\mathbf{2}$ and $\mathbf{3}$ are brought close together in the centre of the supramolecular cavity. In the presence of tripyridyltriazine (6), which binds extremely strongly to 1 via simultaneous coordination of its pyridyl groups to all three zinc atoms ${ }^{3}$, no acceleration of the Diels-Alder reaction was observed. In this capacity, 6 seems to take a role similar to that of a classical competitive enzyme inhibitor.
Although 1 accelerates the above Diels-Alder reaction by several orders of magnitude and is stereoselective for the exo form, the reaction is stoichiometric rather than catalytic. That is, the DielsAlder product binds too strongly to 1 to allow effective turnover.

In exciting work soon to be reported by Sanders's group ${ }^{4}$, a related system in which there is an acyl transfer reaction between two substrates bound in the cavity of 1 has been shown to be catalytic. In this case, the catalytic behaviour reflects the fact that only the reaction intermediate is strongly bound in the cavity.

The analogous cyclic dimer and tetramer corresponding to 1 have also been synthesized $^{5}$, giving access to a range of cavity sizes and relative orientations of the substrate binding sites for use in future studies. Clearly, cyclic porphyrin species such as these have the potential to catalyse different reaction types involving substrates of different sizes. The function of the cyclic cavity is mainly concerned with substrate recognition and orientation and the versatility of the overall system is increased through both of these being effectively decoupled from the reaction site. A further refinement of these systems seems feasible to give the prospect of 'tuning' the binding strength of particular guests by incorporating electron-pushing (or electron-pulling) substituents on the outside of the cavity. In this manner more deliberate control of potential catalytic behaviour may be possible.

The synthesis of the cyclic species is interesting in its own right, as higher yields were obtained when the respective ringclosing reactions were done in the presence of suitable template molecules ${ }^{4}$. For the production of $\mathbf{1}$, tripyridyltriazine (6) was used as the template, because its size and shape are nicely complementary to the cavity in the cyclic trimer.

Although the field of synthetic enzymes remains fairly rudimentary - especially when the extraordinarily high catalytic efficiencies of the natural systems are considered - steady progress is being made. If supramolecular chemistry continues to develop as rapidly as at present, considerably improved synthetic enzymes should emerge in the foreseeable future.

Leonard F. Lindoy is in the Department of Molecular Sciences, James Cook University, Townsville 4811, Queensland, Australia.

\footnotetext{
1. Anderson, H. L., Bashall, A. Henrick, K., McPartlin, M. \& Sanders, J. K. M. Angew. Chem. int. Edn engl. 33 Sanders, J. K. M. An

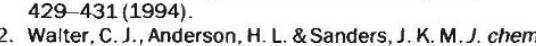
Soc. chem. Commun. 458-460 (1993).

3. Anderson, H. L. \& Sanders, J. K. M. J. chem. Soc. chem. Commun. 1714-1715 (1989).

4. Mackay, L. B., Wylie, R. S. \& Sanders, J. K. M. J. Am. chem Soc. (in the press)

5. Anderson, S., Anderson, H. L. \& Sanders, J. K. M. Accts chem. Res. 26, 469-475 (1993).
} 